\title{
Enhanced recency effects with changing-state and primary-linguistic stimuli
}

\author{
HOWARD J. KALLMAN and PATRICIA CAMERON \\ State University of New York, Albany, New York
}

\begin{abstract}
A number of explanations for the modality effect in immediate serial recall have been proposed. The auditory advantage for recall of recency items has been explained in terms of (1) the contributions of precategorical acoustic storage (PAS), (2) an advantage of changing-state over static stimuli, and (3) an advantage of primary-linguistic coding. Four experiments were conducted to evaluate these hypotheses. In the first, subjects viewed seven consecutive rectangles of different colors on a computer monitor. A small recency effect was obtained when the task was to recall the colors of the rectangles in order, with the size of the effect being independent of whether the rectangles remained stationary on the screen or moved in one of four directions. However, when the task was to recall the direction of movement of the rectangles, a larger recency effect was found. This pattern of results was interpreted as suggesting that recency effects are enhanced by changing-state stimulus information, but only when the changing-state information serves to identify the stimulus. Experiments 2 and 3 provided converging evidence by demonstrating an analogous recency advantage for changing-state visual stimuli that were somewhat different from those of Experiment 1. Experiment 4 demonstrated recency effects with synthesized speech stimuli that were substantially greater than were those found with the changing-state visual stimuli of the first three experiments. Implications of the results for the PAS, changing-state, and primary-linguistic hypotheses, as well as temporal-distinctiveness theories of recency, are discussed.
\end{abstract}

When subjects perform immediate serial recall of lists that are presented auditorily, recency effects are found. In contrast, if the lists are presented visually, recency is usually small or nonexistent. Crowder and Morton (1969) explained this modality effect by assuming that, with auditory presentations, information about the last list item is maintained in precategorical acoustic storage (PAS), and that PAS persists longer than does the corresponding visual storage. Accordingly, auditory presentation leads to more accurate recall of the last item of a list, because information held in PAS, as well as in other memory stores, can be used to aid recall. However, if the last item of an auditory list is followed by an auditory suffix (i.e., a redundant auditory item that is not to be recalled), recency diminishes or disappears altogether, presumably because the suffix masks the last list item in PAS (Crowder, 1978). There are then two central components of the PAS account of this modality effect: (1) auditory storage persists longer than does visual storage and, (2) an auditory suffix interferes with recall of the last item of an auditory list.

Recent findings have challenged Crowder and Morton's PAS explanation of modality effects by demonstrating that robust recency and suffix effects can be found with well-

We thank Deborah Becker, Deborah Newland, and Frank Casdia for help in conducting the experiments and data analysis, and Shirley Brown for her comments on an earlier version of this article. Correspondence may be sent to Patricia Cameron, 12 Briggs Court, Queensbury, NY 12804. chosen visual stimuli. For example, if subjects lip-read rather than hear the items of a list (Campbell \& Dodd, 1980 ), or silently articulate the items of a visually presented orthographic list (Greene \& Crowder, 1984; Nairne \& Walters, 1983), recency effects obtain. Furthermore, a lip-read suffix interferes with recall of items that have been presented auditorily (Spoehr \& Corin, 1978), and silently articulating a suffix interferes with recall of visually presented items that have been recited aloud by subjects (Nairne \& Crowder, 1982), or silently articulated (Nairne \& Walters, 1983).

Three explanations for these findings have received recent attention. The modified-PAS explanation (Crowder 1983, 1986; Greene \& Crowder, 1984) continues to highlight the contribution of PAS, but assumes that, in addition to activation by auditory input, features in PAS may be activated by certain nonauditory events, such as silent articulation of speech, or observation of speech gestures, for example, in lipreading.

A second view is that suffix and enhanced recency effects occur when list items are presented in a primarylinguistic code, that is, one that does not need recoding in working memory (Shand \& Klima, 1981). For example, the physical attributes of a spoken word directly specify the corresponding verbal code in working memory. Accordingly, Shand and Klima suggest that "native speakers of a single speech community share a common phonological code as a primary linguistic code" (p. 466). Presumably, such a code is conveyed by lipread or mouthed stimuli as well as by purely auditory 
stimuli. In contrast, visually presented orthographic stimuli are assumed to undergo recoding in working memory into a format that is not tied directly to the physical attributes of the stimulus. According to the primarylinguistic view, then, enhanced recency is thought to reflect an advantage of primary-linguistic input rather than an advantage of PAS over visual storage. A limitation of the primary-linguistic theory is that it cannot explain cases of enhanced recency that have been demonstrated with stimuli, such as notes played on a piano, that presumably do not directly access the primary-linguistic system (e.g., Greene \& Samuel, 1986).

The third view of modality and suffix effects draws on a distinction between static and changing-state stimuli (Campbell \& Dodd, 1980; Campbell, Dodd, \& Brasher, 1983). Typical auditory stimuli change dynamically during presentation. For example, the formants of a speech sound change during articulation. As another example, changes in fundamental frequency within a sentence provide information about intonation. In contrast, the sorts of visual stimuli typically used in memory experiments, such as written words, remain static throughout presentation. Campbell and Dodd suggested that changing-state stimuli yield enhanced recency, whereas static stimuli do not. According to this view, the advantage of auditory over visual presentations in producing recency effects should disappear with the use of visual stimuli that incorporate changing-state information.

One of the difficulties involved in testing these alternative explanations of recency effects is that they often make the same predictions. For example, spoken words are both primary-linguistic and changing-state stimuli and, furthermore, are assumed to activate features in auditory storage. Clearly then, the demonstration of recency effects with lists of spoken words cannot be used to choose among the three explanations of recency. On the other hand, visually presented words are neither primarylinguistic nor changing-state stimuli, and they would not be expected to activate features in PAS. Therefore, failure to find recency with written words cannot be used to support one view of recency over the others. Finally, lipread words represent both primary-linguistic and changing-state stimuli. There is evidence that such stimuli may activate representations of auditory features (MacDonald \& McGurk, 1978; McGurk \& MacDonald, 1976), conceivably in PAS (Crowder, 1986). So recency with lip-read stimuli does not help much in testing among the three hypotheses.

This is not to say that there is a complete absence of data bearing on the relative merits of the modified-PAS, primary-linguistic, and changing-state hypotheses. Crowder (1986) conducted a series of experiments designed, among other things, to evaluate the changing-state hypothesis. In the relevant experiments, the task was to recall in order a list of digits that had been presented visually on a CRT. In one of the experiments (Experiment 3), each stimulus consisted of a numeral painted slowly on a CRT such that the defining features of each numeral appeared gradually, one after the other. In two additional experiments, the digits were presented orthographically, but the letters of each digit word were presented one followed by the next, from left to right. In Experiment 4, the first letters of each word remained on display as the later letters were revealed (e.g., F, followed by FO, FOU, and FOUR); then, in Experiment 5, the earlier letters were removed as the later letters were presented (e.g., F, followed by FO, OU, UR, and R). The three experiments failed to show any evidence of enhanced recency in any of the changing-state conditions, leading Crowder to dismiss the changing-state hypothesis. The same conclusion was reached by Manning and Gmuer (1985), based on an experiment in which letters were presented tactually, and by Shand and Klima (1981), who found that static signs presented to native signlanguage readers were as effective in producing recency as were signs that included motion.

Rejection of the changing-state hypothesis may be premature, however. One variable that may be significant in whether stimulus change results in enhanced recency is whether the stimulus change that occurs during presentation conveys information critical to identification of the stimulus. This variable has not, as yet, been systematically examined; however, Nairne (1988) recently suggested that task demands, and their effect on the features of a stimulus that are attended to, may be significant in determining whether enhanced recency effects obtain.

For example, in Crowder's (1986) experiment in which numerals were gradually revealed, the change in the stimulation did not define the identity of the stimulus. Whether a particular numeral was a 1,2 , or 3 , and so forth, was conveyed by the particular visual features that were presented on the CRT, not by the pattern of change in what was presented. A different temporal order of occurrence of the features would have modified the patterns of change within a stimulus, but not the identity of the numeral itself. Indeed, from the subject's standpoint, it probably would have been best to have ignored the pattern of change while focusing on integrating the visual features into a steady-state image of the presented stimulus. For these reasons, the changing-state information could be considered task-irrelevant, because, for purposes of the experimental task, it did not define the identity of the stimulus, and its registration was therefore not critical to task performance. The same argument applies to Crowder's orthographic displays. Although Crowder's study suggests that stimulus change per se may not lead to enhanced recency, it leaves open the possibility that task-relevant stimulus change (i.e., change that defines the identity of a stimulus) does. Our present experiments, therefore, took, as a point of departure, the distinction between task-relevant and -irrelevant stimulus change.

\section{EXPERIMENT 1}

In Experiment 1, we contrasted three conditions. In all conditions, seven consecutive color patches were displayed on a computer monitor, and the task was to recall 
the stimuli in order. In the first condition (stationary/report color), the color patches remained stationary during their presentations, and the subject was to recall the colors of the seven patches. The second condition (moving/report color) was similar, except that the patches moved in one of four directions during their presentations. Although the stimuli moved during their presentations, the movement was irrelevant to the recall task because the subjects had only to report the colors. If stimulus change per se were important to the recency effect, greater recency would be expected in the moving/report-color condition than in the stationary/report-color condition. In the final condition (moving/report direction), the stimuli were the same as in the second condition, but the task was to recall the direction of movement of each of the seven stimuli. In this condition, then, the changing state information was task-relevant. To the extent that the task relevance of the changing-state information is important to recency, it would be expected that recency would be most pronounced in this last condition.

Admittedly, our view of what constitutes a changingstate stimulus appears to differ somewhat from Campbell et al.'s (1983). They suggested that a significant characteristic of a changing-state stimulus is that the features "unfold" in time such that the later features within a stimulus could mask the earlier ones. In contrast, we have interpreted changing-state stimuli more generally to mean any stimulus that undergoes detectable change during its presentation. The moving stimuli that we used fit into this broader definition, but may differ from what Campbell et al. had in mind. Nevertheless, if we should demonstrate enhanced recency with our stimuli, this finding would suggest that the unfolding of features may not be the critical factor in those cases in which changing-state stimuli have yielded enhanced recency.

\section{Method}

Subjects. The subjects were 24 undergraduates at the State University of New York at Albany, who participated to earn credit in an introductory psychology course. All reported that they had normal color vision.

Apparatus and Procedure. The stimuli were produced using a Commodore 64 computer system and Commodore 1902 color monitor. A trial consisted of seven consecutively presented colored rectangles, each presented for $700 \mathrm{msec}$. Each rectangle was filled with one of four solid colors (red, green, yellow, or blue), and was $3.5 \mathrm{~cm}$ wide and $3.8 \mathrm{~cm}$ high. At the onset of presentation, the rectangle was presented in the center of the monitor screen, which was $27 \mathrm{~cm}$ wide and $19.5 \mathrm{~cm}$ high. In the stationary/report-color condition, the rectangles remained centered on the screen during the entire presentation time while the rest of the screen was gray. The offset of one rectangle was separated by $300 \mathrm{msec}$ from the onset of the next, and the entire screen was gray during this interstimulus interval. The colors of the rectangles varied randomly from stimulus to stimulus, with the restriction that no two consecutive rectangles within a trial could be the same color.

In the moving/report-color and the moving/report-direction conditions, the seven colored rectangles were also presented for $700 \mathrm{msec}$ each, with 300 -msec interstimulus intervals. However, during the 700-msec display time, the rectangle moved at a constant rate in one of four directions: up, down, left, or right. When moving up or down, the rectangle moved a total distance of $4.5 \mathrm{~cm}$, and when moving left or right, the distance was $3.8 \mathrm{~cm}$. In addition to randomizing the color of each stimulus (with the same restriction as in the stationary condition), the direction of travel of each stimulus was randomly determined, with the additional restriction that no two consecutive rectangles within a trial could travel in the same direction.

In the first two conditions, the task was to recall the colors of the seven rectangles in order. In the third condition (moving/report direction), the task was to recall the direction of movement of the seven rectangles in order. An answer sheet was provided with seven blanks per trial. The subject was instructed to write the first letter of each color or direction in the appropriate blank, to guess if unsure, and to recall from left to right (first item to last) on the answer sheet; recall was self-paced.

There were a total of 21 trials within each block. The first three trials were used for practice, and the task for the first two was to recite out loud the color or direction of movement of each rectangle as it was presented. This was done to familiarize the subject with the stimuli and to verify that the subject could differentiate among them. Following these two trials, 19 memory trials were presented with the first excluded from data analysis.

Each subject was tested on three blocks of trials, with each block representing a different condition. There were six possible orderings of the three types of trial blocks, with 4 subjects tested at each ordering.

Testing was done in an IAC soundproof booth with normal illumination. The subject sat approximately $50 \mathrm{~cm}$ from the monitor screen. The experimenter sat next to the subject to monitor compliance with the recall instructions and to operate the computer.

\section{Results and Discussion}

The mean number of errors (out of a possible 18) at each serial position for the three conditions of Experiment 1 are presented in the left panel of Figure 1. An item was scored as correct only if it was reported in the correct serial position. The right panel of Figure 1 shows the normalized percentages of errors at each serial position, a measure that has often been used to supplement the raw data in immediate-serial-recall experiments. To calculate the normalized percentages, each subject's total number of errors in a particular condition was determined. The percentage of the total errors in the condition that occurred at each serial position was then calculated. The advantage of the normalized measure is that it provides a measure of recency that is relatively independent of both the overall level of performance of individual subjects and the task difficulty.

Analyses of variance were performed on the raw error data and the normalized error data. Conditions ( 3 levels) and serial positions (7 levels) served as within-subjects factors. The degrees of freedom reported with each test reflect the Huynh and Feldt (1976) correction for violations of sphericity.

As expected, the effect of serial position was significant for the raw errors $[F(3.47,79.90)=74.60$, $\left.p<.001, M S_{\mathrm{e}}=6.29\right]$ and for the normalized percentages $[F(2.67,61.45)=57.19, p<.001]$. With the raw error data, the effect of condition was not significant ${ }^{1}$ $\left[F(2,46)=1.89, p>.10, M S_{\mathrm{e}}=10.72\right]$, but the interaction between condition and serial position was $\left[F(9.80,225.44)=2.42, p<.01, M S_{\mathrm{e}}=3.43\right]$. There 

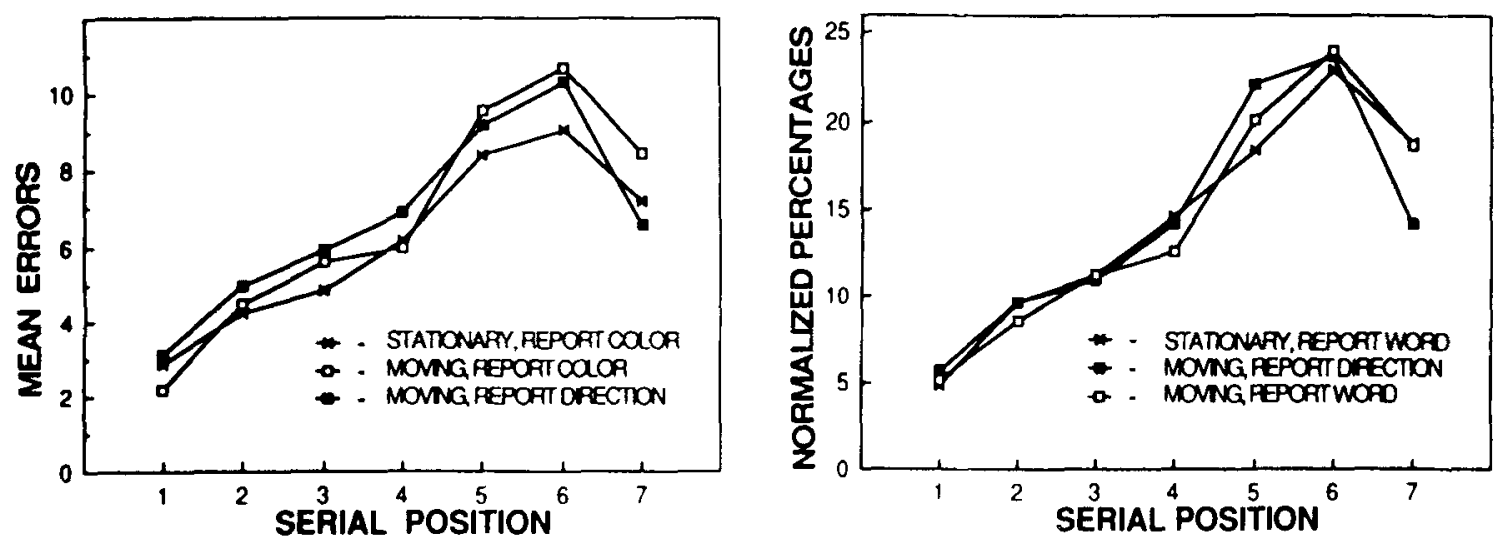

Figure 1. Mean number of errors (out of a possible 18) as a function of serial position for the three conditions of Experiment 1 are presented in the left panel. In the right panel are normalized percentage of errors as a function of serial position for the three conditions of Experiment 1.

was no significant interaction for the normalized data $\left[F(8.17,187.87)=1.68, p>.10, M S_{e}=20.93\right]$.

Because recency in immediate-serial-recall experiments is often assessed by comparing performance at the last serial position to that at the next-to-last serial position, a separate analysis was performed using data from the sixth and seventh serial positions only. A significant effect of conditions on the error data $[F(2,46)=5.65, p<$ $\left..01, M S_{\mathrm{e}}=4.86\right]$ was due primarily to the greater number of errors made in the moving/report-color condition. However, the effect of conditions for the normalized data was not significant $[F(2,46)=2.31, p>.10$, $\left.M S_{c}=28.02\right]$. A significant effect of serial position on both the raw error data and the normalized data $[F(1,23)$ $=71.52, p<.001, M S_{\mathrm{e}}=3.47$; and $F(1,23)=39.39$, $p<.001, M S_{\mathrm{e}}=35.43$, respectively] confirms the presence of a recency effect. The size of the recency effect depended on the condition as indicated by a significant interaction between condition and serial position $\left[F(2,46)=5.43, p<.01, M S_{\mathrm{e}}=2.17\right.$ (raw error data); and $F(1.99,45.82)=5.95, p<.01, M S_{\mathrm{e}}=11.00$ (normalized data)].

There were two specific comparisons involving recency that were of interest. The first was whether the size of recency differed for the stationary/report-color and the moving/report-color conditions. In both conditions, recall of the color information was required, but there was movement of the stimulus in the latter condition only. If stimulus movement per se enhances recency, a difference between these two conditions would be expected. However, the size of the recency effect obtained in the stationary, report-color and the moving/report-color conditions did not differ with either the raw errors $[t(23)=$ $\left.1.05, p>.25, S E_{\mathrm{D}}=.356\right]$ or the normalized percentages $\left[t(23)<1, S E_{\overline{\mathrm{D}}}=1.30\right]$.

The second planned comparison was between the moving/report-color and the moving/report-direction conditions. In both cases, there was stimulus movement, but the stimulus movement was relevant to the task in the last condition only. Recency was, in fact, greater in the moving/report-direction condition than it was in the moving/report-color condition, in terms of the raw errors $\left[t(23)=2.35, p<.05, S E_{\overline{\mathrm{D}}}=.640\right]$ and the normalized percentages $\left[t(23)=3.26, p<.01, S E_{\overline{\mathrm{D}}}=1.20\right]$.

To summarize, Experiment 1 demonstrated a recency effect in immediate serial recall, the size of which depended on testing condition. The greatest recency effect was found with the changing-state stimuli, but only when the task required a report of information that was directly tied to the change. If the stimulus change did not serve to identify the stimulus, recency was comparable to that found with stationary stimuli.

\section{EXPERIMENT 2}

Experiment 1 demonstrated an enhanced recency effect with changing-state stimuli. It could be objected, however, that the recency effect found in the moving/reportdirection condition reflected some factor other than taskrelevant movement. One obvious difference that separated the conditions was that words to be reported in the moving/report-direction condition differed from those to be reported in either of the report-color conditions, and this factor could have somehow affected the results. This confounding was addressed in Experiment 2. Another possibility is that the differences between conditions simply reflected decay-time differences in sensory storage between the color and the movement information in our stimuli. To control for this possibility, the displays in Experiment 2 were all monochrome, and the task was modified accordingly.

An additional concern was that, although the statistical tests of Experiment 1 supported the presence of an enhanced recency effect in the moving/report-direction condition, the size of the effect was not substantial. Accordingly, we felt that it was important to provide additional 
evidence that task-relevant movement could result in an enhanced recency effect.

\section{Method}

Subjects. The subjects were 42 undergraduates at the State University of New York at Albany who participated to earn credit in an introductory psychology course.

Apparatus and Procedure. The design was similar to that of Experiment 1; there were three blocked conditions presented to the subjects in counterbalanced orders. Except where noted below, the procedure (e.g., number of trials, number of stimuli per trial, format of answer sheet) was the same as in Experiment 1.

In all of the conditions of Experiment 2, the subject's task was to view seven consecutive stimuli, each of which could be classified for purposes of report as representing either left, right, up, or down. After viewing the seven stimuli of a trial, the subject was to report them in order, as in Experiment 1 . In the stationary/reportword condition, either the word "left," "right," "up," or "down" was presented in the middle of the screen, enclosed in a rectangle. In the moving/report-word condition, the display was identical to that in the stationary/report-word condition, except that the word and its bordering rectangle moved in one of four diagonal directions (i.e., $45^{\circ}, 135^{\circ}$, etc.) during its presentation. ${ }^{2}$ In the moving/report-direction condition, the rectangle alone was presented, and moved either up, down, left, or right. During its $700-\mathrm{msec}$ presentation, the rectangle moved approximately $3.5 \mathrm{~cm}$ along the horizontal or diagonal axes, or $4.3 \mathrm{~cm}$ along the vertical. The task in each case was to recall the sequence of ups, downs, lefts, and rights, which was conveyed by the words in the first two conditions and by the direction of movement in the third condition.

The rectangle was demarked by black lines. The vertical lines were $3.5 \mathrm{~cm}$ long and the horizontal $2.8 \mathrm{~cm}$. The area inside and outside of the rectangle was light green, while the letters of any words enclosed within the rectangle were black. When a word was presented, the first letter was uppercase and stood $1 \mathrm{~cm}$ high, while the others were lowercase and ranged from 0.6 to $1 \mathrm{~cm}$ high. The dimensions of the monitor screen were approximately $19 \mathrm{~cm}$ high by $25 \mathrm{~cm}$ wide.

\section{Results and Discussion}

The mean number of errors at each serial position for each of the three conditions are presented in the left panel of Figure 2; the normalized percentages are presented in the right panel. Analyses of the data followed the same procedures as for Experiment 1 .

The effect of serial position was significant for the raw errors $\left[F(3.18,130.48)=128.02, p<.001, M S_{\mathrm{e}}\right.$ $=5.92]$ and for the normalized percentages $[F(2.76$, $\left.113.78)=99.01, p<.001, M S_{\mathrm{e}}=53.96\right]$. With the raw error data, the effect of condition was not significant $\left[F<1, M S_{\mathrm{e}}=14.52\right]$. The interaction between condition and serial position was not significant for the raw errors $\left[F(8.57,351.19)=1.51, p>.10, M S_{\mathrm{e}}=3.45\right]$ or for the normalized percentages $[F(7.98,327.03)=$ $\left.1.03, M S_{\mathrm{e}}=27.04\right]$.

The separate analyses of the sixth and seventh serial positions failed to show any significant main effects of conditions $(F<1$ for raw errors and normalized percentages, $M S_{\mathrm{e}}=7.76$ and $M S_{\mathrm{e}}=39.30$, respectively). There was a recency effect, however, as confirmed by a significant effect of serial position [errors: $F(1,41)=50.08$, $p<.001, M S_{\mathrm{e}}=3.73$; normalized percentages: $F(1,41)$ $\left.=32.44, p<.001, M S_{\mathrm{e}}=41.56\right]$. Of primary interest was the interaction between serial position and condition, which was significant for the raw errors $[F(2,82)=4.59$, $p<.02, M S_{\mathrm{e}}=3.31$ ), but was marginal for the normalized percentages $[F(1.89,77.65)=2.40, .05<p<.10$, $\left.M S_{\mathrm{e}}=34.10\right]$.

There were two specific comparisons of interest, as in Experiment $1 .^{3}$ There was a greater recency effect for the moving/report-direction condition than there was for the moving/report-word condition, in terms of raw errors $\left[t(41)=2.66, p<.01, S E_{\overline{\mathrm{D}}}=.60\right]$ and normalized percentages $\left[t(41)=2.54, p<.01, S E_{\overline{\mathrm{D}}}=1.52\right]$. In contrast, there was no difference in the size of the recency effect between the moving/report-word and the stationary/ report-word conditions [raw errors: $t(41)<1, S E_{\overline{\mathrm{D}}}=$ .57; normalized percentages: $t(41)<1, S E_{\overline{\mathrm{D}}}=1.81$ ].

The results of Experiment 2 were in agreement with our prediction: Specifically, an enhanced recency effect, although relatively small in magnitude, was demonstrated for the moving/report-direction condition.
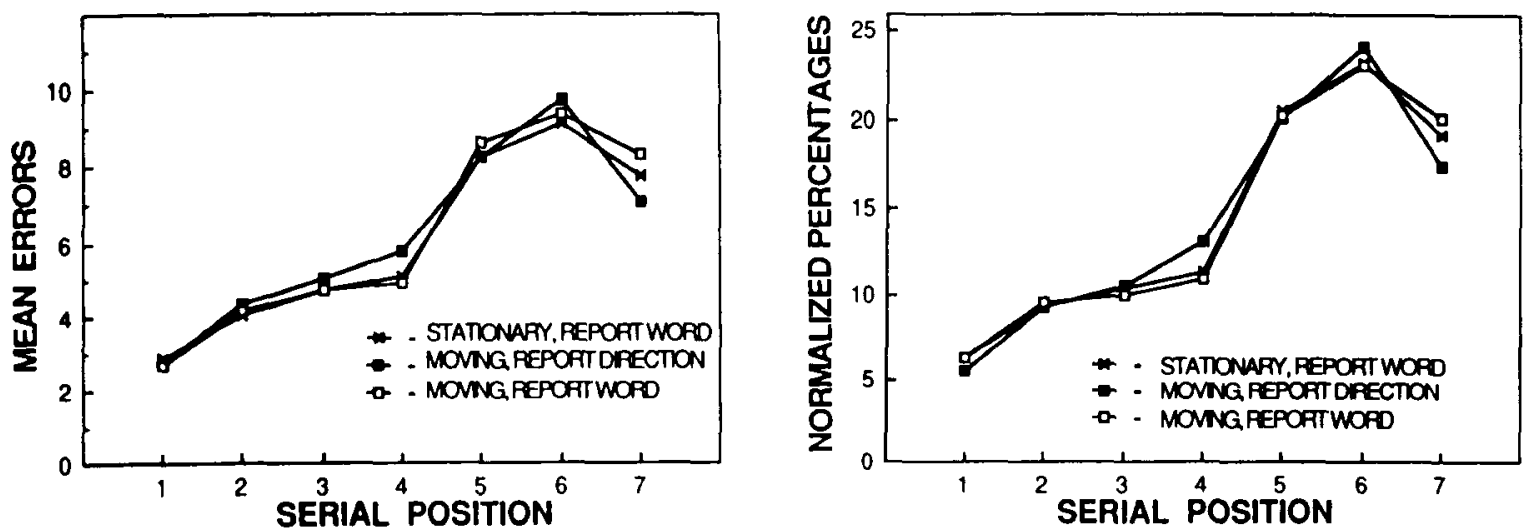

Figure 2. Mean number of errors as a function of serial position for the three conditions of Experiment 2 are presented in the left panel. In the right panel are the normalized percentage of errors as a function of serial position for the three conditions of Experiment 2. 


\section{EXPERIMENT 3}

Although Experiments 1 and 2 demonstrated enhanced recency with changing-state stimuli, categorization of each stimulus could have been based on the subjects' having simply noted that at stimulus offset, the stimulus was below, above, to the right of, or to the left of center. Thus, although the coding of movement would have been sufficient for performance on the memory task, it would not have been necessary. Alternatively, the results might implicate an advantage of location information rather than one of changing-state information in memory. This potential confounding was addressed in Experiment 3 .

As in Experiment 2, the task was to view seven consecutive stimuli and, after presentation, to report the sequence of lefts, rights, ups, and downs. The stimuli and conditions were identical to those of Experiment 2 except that each rectangle was initially presented at one of nine locations on the screen, randomly varied from stimulus to stimulus. Thus, in the moving/report-direction condition, the direction of movement could not be inferred from the final location of the rectangle, and responses would have had to be based on the perceived movement of the stimulus.

\section{Method}

Subjects. The subjects were 42 undergraduates at the State University of New York at Albany who participated for credit in an introductory psychology course.

Apparatus and Procedure. The apparatus and procedure were identical to those of Experiment 2, with one exception: In Experiment 3 , the starting point for each stimulus varied randomly from among nine screen positions (recall that in Experiment 2, each stimulus was initially presented at the center of the screen). The nine possible starting positions formed three rows of three positions. In describing the positions, we will refer to that point at which the center of the rectangle was placed. One of the possible starting positions was at the screen center. Within that same row, possible starting positions also included positions $4 \mathrm{~cm}$ to the left and $4 \mathrm{~cm}$ to the right of center. There were then three starting positions that were $2 \mathrm{~cm}$ above the above-described row, and three that were $2 \mathrm{~cm}$ below the row.

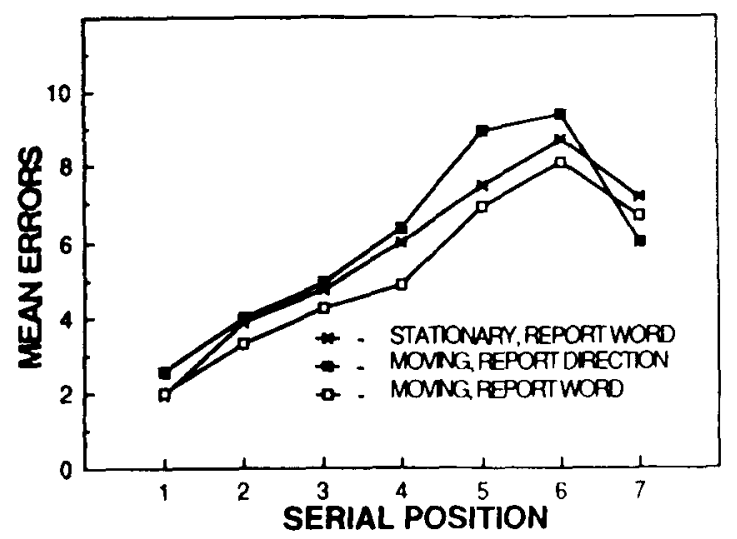

In the stationary/report-word condition, the stimulus remained at the randomly selected position throughout its presentation. In the two moving conditions, the stimulus moved in one of four directions, as in Experiment 2 (that is, up, down, left, or right for moving/report direction, or one of the four diagonal directions for moving/report word, and at the same speed as in Experiment 2.

\section{Results and Discussion}

The mean numbers of errors and the normalized percentages at each serial position for each of the conditions are presented in the panels of Figure 3. The effect of serial position was significant for the raw errors $[F(2.55,104.57)$ $=84.23, p<.001, M S_{\mathrm{e}}=7.951$ and for the normalized errors $\mid F(2.82,115.42)=73.83, p<.001, M S_{\mathrm{e}}=$ 70.20]. The effect of condition was significant for the raw errors $\left.\mid F(2,82)=4.54, p<.02, M S_{\mathrm{e}}=12.14\right]$, with the most errors made in the moving/report-direction condition, followed by the stationary/report-word and the moving/report-word conditions. The interaction between serial position and condition was significant [errors: $F(8.18,335.49)=2.95, p<.01, M S_{\mathrm{e}}=3.64$; normalized percentages: $F(7.22,296.04)=2.09, p<.05$, $M S_{\mathrm{e}}=41.86 \mid$.

The analysis of the sixth and seventh serial positions failed to show an effect of condition [errors: $F<1$, $M S_{\mathrm{e}}=9.79 ;$ normalized percentages: $F(1.77,72.51)=$ $2.31, p>.10, M S_{\mathrm{e}}=57.31 \mathrm{~J}$. The effect of serial position was significant in both cases $[F(1,41)=51.33$, $p<.001, M S_{\mathrm{e}}=5.64 ;$ and $F(1,41)=40.97, p<.001$, $M S_{\mathrm{e}}=63.77$, respectively]. Of primary interest, however, was the significant interaction between condition and serial position [errors: $F(1.77,72.58)=7.18$, $p<.01, M S_{\mathrm{e}}=3.51$; normalized percentages: $F(1.68,68.72)=3.57, p<.05, M S_{\mathrm{e}}=47.19 \mathrm{~J}$. The specific comparison of recency in the moving/reportdirection and the moving/report-word conditions showed a significant difference in terms of errors $[t(41)=2.89$, $p<.01, S E_{\mathrm{D}}=.68 \mathrm{l}$. The normalized percentages analysis showed a marginally significant difference, but only when a one-tailed test was used, $[t(41)=1.67$, $p=.051, S E_{\mathrm{D}}=2.50 \mathrm{~J} \cdot{ }^{4}$ Recency in the moving/

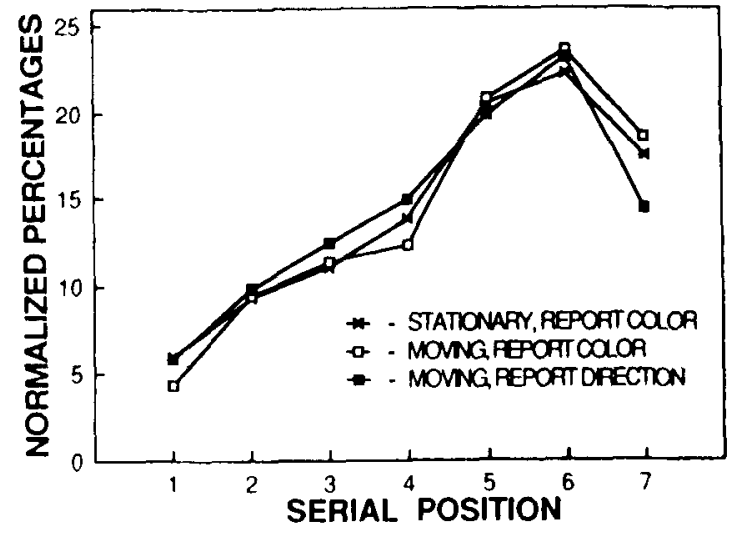

Figure 3. Left panel: Mean number of errors as a function of serial position for the three conditions of Experiment 3. Right panel: Normalized percentage of errors as a function of serial position for the three conditions of Experiment 3. 
report-word and stationary/report-word conditions was essentially equivalent [errors: $t(41)<1, S E_{\overline{\mathrm{D}}}=.48$; normalized percentages: $t(41)<1, S E_{\overline{\mathrm{D}}}=2.18$ ]

The results of Experiment 3 were consistent with our predictions. The greatest recency effect was found in the moving/report-direction condition, while the other two conditions showed smaller effects. These results support our conclusion from Experiments 1 and 2 that recency is enhanced when task-relevant movement is included in the stimulus.

\section{EXPERIMENT 4}

Experiments 1-3 demonstrated enhanced recency effects with changing-state stimuli. It is noteworthy that neither the PAS nor the primary-linguistic positions can explain this enhanced recency effect. Nonetheless, the support provided for some sort of a changing-state explanation by the experiments does not preclude contributions from the primary-linguistic system or PAS to recency in other situations. Indeed, recency effects with speech stimuli tend to be of greater magnitude than were those that we obtained with the changing-state stimuli of Experiments 1-3. However, due to the use of different experimental designs, procedures, and subject pools, it is difficult to compare the size of our effects to those obtained with speech in other studies. Accordingly, one purpose of Experiment 4 was to evaluate the size of recency, using speech stimuli with an experimental design and subject population that were essentially identical to that of Experiments 1-3.

The first two conditions of Experiment 4 involved the presentation of lists of either color words (red, green, yellow, and blue) or direction words (up, down, right, and left) produced by a speech synthesizer. These conditions provided a measure of recency, using speech stimuli words that were identical to those that were to be reported in Experiments 1-3.

The third condition consisted of presentation of a series of seven "moving" tones per trial. Each tone either increased in pitch, decreased in pitch, moved from the middle of the head to the right ear, or moved from the middle of the head to the left ear. The task was to recall the direction of tonal movement-up, down, left, or right-for the seven tones of a trial. This task was designed to be an approximate auditory analogue of the moving/report-directions tasks of the earlier experiments. It might be objected that the actual physical movement of the tones differed in a qualitative way from the movement of our visual stimuli in the earlier experiments; nevertheless, whether the tone was to be classified as moving up, down, left, or right was conveyed by the change in the stimulus. To the extent that auditory presentations offer a recency advantage over and above that provided by changing-state stimuli, recency in the moving-tones condition of Experiment 4 might be expected to be greater than that in the moving/report-direction conditions of Experiment 1-3.
Comparison of the word conditions to the moving-tone condition of Experiment 4 would provide at least a crude test of the contribution of the primary-linguistic system to recency; while both types of stimuli were auditory and included changing-state information, only the words could be expected to tap directly the primary-linguistic coding system.

\section{Method}

Subjects. The subjects were 24 undergraduates at the State University of New York at Albany who participated for credit in an introductory psychology course. All subjects reported having normal hearing.

Apparatus and Procedure. The design was similar to that of Experiments 1-3: there were three blocked conditions presented to the subjects in counterbalanced orders. In the moving-tone condition, seven consecutive tones were presented, after which the listener recalled the direction of movement of the seven in order. The tones were generated by a Coulbourn Instruments Model S2405 voltage-controlled oscillator and Model S77-08 programmable amplifier/digital-to-analog converter, which were controlled by a Commodore 64 computer equipped with a Schnedler Systems interface board. Each stimulus tone had a duration of $250 \mathrm{msec}$. Rise and fall times ( $5 \mathrm{msec}$ each, except as noted otherwise) for the tones were controlled by Coulbourn Instruments Model S84-04 rise/fall gates. The starting frequency of each tone was $740 \mathrm{~Hz}$.

Tones that moved from the center of the listener's head to the right or left ear were at onset, presented binaurally with equal intensity to both ears. Immediately after the onset of the tone at both ears, the tone at one ear was gated off with a linear fall time of $250 \mathrm{msec}$; at the other ear, the tone remained at full amplitude until $5 \mathrm{msec}$ before its offset, at which time a 5 -msec fall was introduced. The frequency of the tone remained constant at $740 \mathrm{~Hz}$ throughout its presentation. The resulting perception was of a fixedfrequency tone that moved either left or right.

Tones that were intended to move either up or down were presented binaurally with equal intensity at both ears. To institute the frequency change, the voltage that controlled the oscillator was gradually increased or decreased, using a Coulbourn Instruments Model S76-01 contour following integrator. The resulting tone either gradually increased from 740 to $1070 \mathrm{~Hz}$ or decreased from 740 to $410 \mathrm{~Hz}$ during its $250-\mathrm{msec}$ duration. The seven tones of a trial were presented at the rate of one per second. Consecutive tones were separated by $750 \mathrm{msec}$ of silence.

The verbal stimuli used in the spoken-color and spoken-direction conditions were generated by a Commodore Magic Voice Speech Module controlled by a Commodore 64 computer. The Magic Voice synthesizer generates speech that resembles a female voice and, although slightly distorted, is easy to understand. In the spokencolor condition, the same colors that were used in Experiment 1 were produced by the synthesizer, with the restriction that the same color word could not be presented as consecutive stimuli within a trial. In the spoken-direction condition, the four directions (up, down, left, and right) were spoken, again with the restriction that no two consecutive words on a trial could be the same. The words were presented at the rate of one per second with an average word duration of approximately $265 \mathrm{msec}$, and silence separated one word from the next.

The auditory stimuli were recorded on cassette tape, using an Aiwa ADF-770 cassette deck. During the experiment, the stimuli were played back on the cassette deck and were presented to listeners at a comfortable listening level through Sony MDR-S50 headphones. During the first two trials of a block, the listener was given a list of the seven stimuli and was told simply to listen as they were played. This procedure was designed to familiarize the listeners with the stimuli. Then, one practice recall trial was followed by $18 \mathrm{ex}-$ 
perimental trials. The recall task and answer sheets were the same as those in Experiments 1-3. The experimenter sat next to the subject to monitor compliance with the recall instructions and to operate the tape deck via a remote control switch (the tape deck itself was in another room to isolate any mechanical noises produced by the deck). Testing was conducted in a sound-attenuated room.

\section{Results and Discussion}

As in Experiments 1-3, separate analyses were conducted on the raw error data and the normalized percentages. The raw error data are presented in the left panel of Figure 4 and the normalized percentages in the right panel.

A significant effect of condition for the raw error data $\left[F(1.92,44.26)=8.48, p<.001, M S_{\mathrm{e}}=22.08\right]$ reflected that there were more errors made in the movingtones condition than there were in the other conditions. The effect of serial position also was significant [raw errors: $F(3.72,85.63)=82.84, p<.001, M S_{\mathrm{e}}=5.76$; normalized percentages: $F(3.25,74.73)=69.79$, $\left.p<.001, M S_{\mathrm{e}}=49.55\right]$. There was a significant interaction between condition and serial position [raw errors: $F(9.75,224.36)=4.66, p<.001, M S_{\mathrm{e}}=3.52$; normalized percentages: $F(8.58,197.26)=4.68, p<.001$, $\left.M S_{\mathrm{e}}=27.11\right]$.

Examination of the sixth and seventh serial positions showed that there were recency effects for all three conditions, but these effects were much greater for the two spoken-word conditions. The two analyses of variance conducted on the data from the sixth and seventh serial positions confirmed the reliability of these trends. The main effect of condition was significant [raw errors: $F(2,46)=21.44, p<.001, M S_{\mathrm{e}}=9.58 ;$ normalized percentages: $\left.F(2,46)=7.22, p<.01, M S_{\mathrm{e}}=33.26\right]$. The main effect of serial position also was significant [raw errors: $F(1,23)=122.22, p<.001, M S_{\mathrm{e}}=4.55$; normalized percentages: $F(1,23)=107.62, p<.001$, $\left.M S_{\mathrm{e}}=42.46\right]$. Finally, the interaction between these two variables was significant [raw errors; $F(2,46)=4.35$, $p<.02, M S_{\mathrm{e}}=3.83$; normalized percentages: $F(2,46)$ $\left.=7.70, p<.01, M S_{\mathrm{e}}=33.21\right]$.

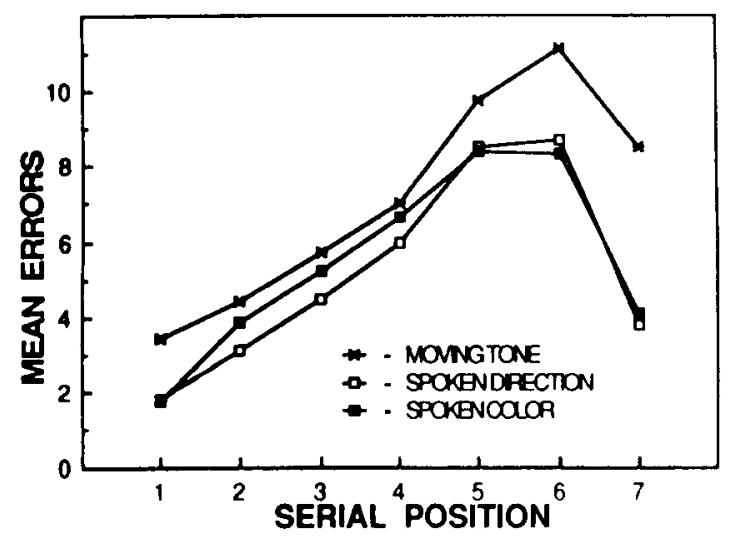

Two planned $t$ tests were conducted to evaluate differences in the size of recency between conditions. The first comparison was between the moving-tone and the spokendirection conditions. In both, the task required that a report of directions be given, but the stimuli conveying the directional information differed. The size of the recency effect was greater with the spoken stimuli than it was with the tonal stimuli [raw errors: $t(23)=2.72$, $p<.05, S E_{\overline{\mathrm{D}}}=.842$; normalized data: $t(23)=4.02$, $\left.p<.001, S E_{\overline{\mathrm{D}}}=2.22\right]$. The $t$ tests showed no difference between recency in the spoken-color and the spokendirection conditions [raw errors: $t(23)=1.07, p>.25$, $S E_{\overline{\mathrm{D}}}=.625$; normalized data: $t(23)=1.08, p>.25$, $\left.S E_{\overline{\mathrm{D}}}=2.30\right]$.

The recency effects in Experiment 4 with verbally presented words were greater than those in any of the other conditions tested in the present series of experiments. In contrast, recency in the moving-tone condition appeared to be of the same magnitude as it was in the demonstrations of recency with other task-relevant moving stimuli in Experiments 1-3.

\section{GENERAL DISCUSSION}

The present results suggest that recency in immediate serial recall is enhanced if stimuli that include taskrelevant changing-state information are presented. The demonstration of enhanced recency in this case suggests that task-relevant movement may be at least as important in demonstrating recency as is the unfolding of features over time. However, the size of the recency effect found in the moving/report-direction conditions was less than that usually found when lists of items are presented verbally, a point reinforced by the spoken-word condition of Experiment 4. Thus, although our data provide support for the importance of the changing-state variable, as we have defined it, it appears that other factors may also contribute to enhanced recency.

The finding that recency found in the spoken-word conditions was greater than that found in any of the others

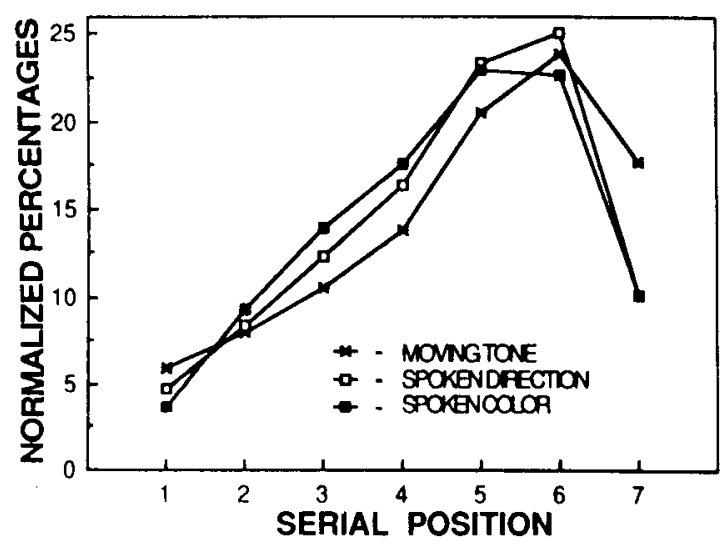

Figure 4. Left panel: Mean number of errors as a function of serial position for the three conditions of Experiment 4. Right panel: Normalized percentage of errors as a function of serial position for the three conditions of Experiment 4. 
is consistent with, although not in itself unequivocal evidence for, a role of primary-linguistic coding in enhanced recency. One of the difficulties in providing unequivocal support for the primary-linguistic hypothesis is that a rigorous test requires a comparison of primary-linguistic stimuli to stimuli that differ by the single dimension of code type. If other dimensions covary, as is often the case, it is difficult to isolate the contribution of primarylinguistic coding. Although the size of the recency effect in the spoken-direction condition of Experiment 4 exceeded that in the moving/report-direction conditions of Experiments 1,2, and 3, this comparison must be viewed with caution, because the changing-state information in the two conditions was not equated in terms of complexity of stimulus change. The stimulus change that occurred in the moving/report-direction condition followed a single trajectory, while the acoustic changes that characterized the spoken words were considerably more complex. At present, we can only speculate as to whether complexity of stimulus change affects recall of recency items, and so, we cannot rule out that stimulus-change complexity, rather than code type, might have been responsible for the difference between the spoken-direction and the moving/report-direction conditions.

The PAS view fared the worst in our tests. The movingtone and the moving/report-direction conditions yielded comparable recency effects, a finding that appears to run counter to PAS theory. In both conditions, the stimulus change could be described by a single trajectory, and the task was to report the directions of the stimuli. PAS theory predicts an advantage for auditory over visual presentations, and, thus, an advantage for the moving-tone condition, a result that did not materialize. Admittedly, the comparison was not entirely clear-cut, given that the durations of the visual and auditory stimuli differed. ${ }^{5}$ However, we doubt that this variable contributed to the failure to find differences between the visual and auditory stimuli. The spoken words, which had durations averaging $265 \mathrm{msec}$, yielded the greatest recency of all the conditions that we tested. This, along with the fact that the 250-msec durations of the tones allowed more than enough time for the registration of the changing-state tonal information, suggests that the 250 -msec durations were not a limiting factor in producing recency.

To summarize, while the present experiments support the idea that presentation of stimuli that include taskrelevant, changing-state information can lead to enhanced recency effects, there is nothing in our results to contradict the possible role of primary-linguistic coding in some recency effects. Finally, our results are somewhat disparaging to the PAS theory, although our test of this was rather limited.

In contrast to the hypotheses considered thus far, it might be that the enhanced recency effects found in various immediate-serial-recall tasks, including our own, stem from some fundamental property of the memoryprocessing system, and, consequently, the findings that have been used as support for either changing-state, primary-linguistic, or PAS hypotheses could all be explained by a single principle. Such a view would not negate the value of isolating the types of stimuli-auditory, changing-state, primary-linguistic, and so forth-that yield enhanced recency effects. But, in addition, it would be of interest to show how these various classes of stimuli are related by some general principle. Along these lines, Gardiner (1983) and Glenberg and Swanson (1986) have developed temporal-distinctiveness theories that propose, among other things, to explain recency and modality effects in immediate recall.

According to the temporal-distinctiveness view, factors that enhance the temporal discriminability of list items in memory should contribute particularly to enhanced recall of recency items. Glenberg and Swanson (1986) presented a model of retrieval that is based on the idea that the temporal context of an item serves as a recall cue. Although the reader is referred to their article for details, it is sufficient here to note that, to the extent that the time of presentation of recency items is encoded with a fine grain, their model predicts that recall of the recency items should be particularly good because the recency items would then be distinct in memory.

Converging evidence for a temporal-distinctiveness theory of recency comes from a study that showed that temporal information is more faithfully represented given verbal rather than orthographic item presentations (Metcalfe, Glavanov, \& Murdock, 1981). Although this study has been interpreted as showing that the auditory modality offers finer grain temporal encodings than the visual modality, it might be more prudent to conclude that the temporal characteristics of typical auditory stimuli are better represented in memory than are the temporal characteristics of typical visual stimuli. Whether this difference between stimuli is due directly to the modality variable, or to some other variable that is correlated (e.g., changingstate or primary-linguistic coding), is left unanswered and worthy of future research. Certainly, demonstrations of enhanced temporal coding of the various types of stimuli that yield enhanced recency effects would strengthen considerably the viability of temporal-distinctiveness theories of recency.

For example, it would be useful to determine whether task-relevant stimulus change enhances the encoding of the temporal aspects of a stimulus. Although we cannot offer direct evidence to support this, analysis suggests that the proposition is reasonable. Because task-relevant stimulus change would need to be encoded for purposes of stimulus identification, the changing-state information within a stimulus would represent an additional element to which the temporal context could become associated. On the other hand, if the changing-state information were irrelevant to stimulus identification, there would be little reason for encoding the change, and, hence, the contribution to temporal encoding would be less likely.

A temporal-distinctiveness theory might also be able to accommodate any advantages of primary-linguistic stimuli. One of the characteristics of primary-linguistic 
stimuli is that their physical characteristics bear a nonarbitrary relationship to the corresponding code in working memory. It is thus likely that primary-linguistic stimuli would be encoded rapidly and relatively automatically, and, as a result, there would be little temporal variability associated with the process of encoding the stimulus. Given evidence that the encoding of the temporal aspects of a stimulus depend not only on the stimulus' duration, but also on the amount of time spent on the processing of nontemporal stimulus information (Cantor \& Thomas, 1976; Thomas \& Brown, 1974; Thomas \& Cantor, 1975, 1978; Thomas \& Weaver, 1975), the rapid and relatively automatic encoding of primary-linguistic stimuli would be apt to result in a fine-grained representation of temporal stimulus information. In contrast, this expectation would not hold for stimuli whose physical characteristics bear an arbitrary relationship to the corresponding code in working memory, because an additional transformation would be required for encoding, and this additional time and associated variability would be added to the encoding process. The greater amount of temporal variability associated with such stimuli could act against a finegrained encoding of the temporal context and, consequently, diminish the likelihood of recency effects.

At this point, it is not clear whether temporaldistinctiveness theory or a combination of the other theories of enhanced recency will ultimately prevail as an explanation for modality effects and other instances of enhanced recency. Temporal-distinctiveness theory offers the advantage of parsimony. Future research, some of which has been suggested in this article, should help establish the validity of a temporal-distinctiveness theory of recency.

\section{REFERENCES}

Campbell, R., \& Dodd, B. (1980). Hearing by eye. Quarterly Journal of Experimental Psychology, 32, 85-99.

CAmpbell, R., Dodd, B., \& Brasher, J. (1983). The sources of visual recency: Movement and language in serial recall. Quarterly Journal of Experimental Psychology, 35A, 571-587.

Cantor, N. E., \& Thomas, E. A. C. (1976). Visual masking effects on duration, size, and form discrimination. Perception \& Perception, 19, 321-327.

CROWDER, R. G. (1978). Mechanisms of auditory backward masking in the suffix effect. Psychological Review, 85, 502-524.

CrowDer, R. G. (1983). The purity of auditory memory. Philosophical Transactions of the Royal Society, 302, 251-265.

Crowder, R. G. (1986). Auditory and temporal factors in the modality effect. Journal of Experimental Psychology: Learning, Memory, \& Cognition, 12, 268-278.

Crowder, R. G., \& Morton, J. (1969). Precategorical acoustic storage (PAS). Perception \& Psychophysics, 5, 365-373.

Gardiner, J. M. (1983). On recency and echoic memory. Philosophical Transactions of the Royal Society of London, 302, 267-282.

Glenberg, A. M., \& Swanson, N. G. (1986). A temporal distinctiveness theory of recency and modality effects. Journal of Experimental Psychology: Learning, Memory, \& Cognition, 12, 3-15.

Greene, R. L., \& Crowder, R. G. (1984). Modality and suffix effects in the absence of auditory stimulation. Joumal of Verbal Leaming \& Verbal Behavior, 23, 371-382.
Greene, R. L., \& Samuel, A. G. (1986). Recency and suffix effects in serial recall of musical stimuli. Journal of Experimental Psychology: Learning, Memory, \& Cognition, 12, 517-524.

HUYNH, H., \& FELDT, L. S. (1976). Estimation of the Box correction for degrees of freedom from sample data in the randomized block and splitplot designs. Journal of Educational Statistics, 1, 69-82.

MacDonald, J., MCGurk, H. (1978). Visual influences on speech perception processes. Perception \& Psychophysics, 24, 253-257.

MANning, S. K., \& Gmuer, B. A. (1985). Visual suffix effects on the Optacon: A test of changing state, primary linguistic, and attentional theories. Bulletin of the Psychonomic Society, 23, 1-4.

MCGURK, H., \& MACDONALD, J. (1976). Hearing lips and seeing voices. Nature, 264, 746-748.

Metcalfe, J., Glavanov, D., \& Murdock, M. (1981). Spatial and temporal processing in the auditory and visual modalities. Memory \& Cognition, 9, 351-359.

NAIRNE, J. S. (1988). A framework for interpreting recency effects in immediate serial recall. Memory \& Cognition, 16, 343-352.

NAIRNE, J. S., \& CROWDER, R. G. (1982). On the locus of the stimulus suffix effect. Memory \& Cognition, 10, 350-357.

NAIRNE, J. S., \& Walters, V. L. (1983). Silent mouthing produces modality- and suffix-like effects. Journal of Verbal Learning \& Verbal Behavior, 22, 475-483.

SHAND, M. A., KLima, E. S. (1981). Nonauditory suffix effects in congenitally deaf signers of American Sign Language. Journal of Experimental Psychology: Human Learning \& Memory, 7, 464-474.

SPOEhr, K. T., \& Corin, W. J. (1978). The stimulus suffix effect as a memory coding phenomenon. Memory \& Cognition, 6, 583-589.

Thомas, E. A. C., \& Brown, I. (1974). Time perception and the filledduration illusion. Perception \& Psychophysics, 16, 449-458.

Thomas, E. A. C., \& Cantor, N. E. (1975). On the duality of simultaneous time and size perception. Perception \& Psychophysics, 18, 44-48.

Thomas, E. A. C., CANTor, N. E. (1978). Interdependency between the processes of temporal and non-temporal information. In J. Requin (Ed.), Attention and performance VII. Hillsdale, NJ: Erlbaum.

Thomas, E. A. C., Weaver, W. B. (1975). Cognitive processing and time perception. Perception \& Psychophysics, 17, 363-367.

\section{NOTES}

1. The effect of condition on the normalized percentages is meaningless due to the fact that the normalized percentages for a given condition must sum across serial positions to 100 . Rounding errors alone contribute to differences between the different conditions, and, thus, this factor in the analysis will go unreported.

2. We used diagonal movement instead of horizontal/vertical movement in the moving/report-word condition to minimize any response conflict between the direction of movement and the displayed word. For example, some conflict might be expected if the word "up" were displayed while the rectangle moved downward. Such response conflict would be less likely if the word "up" were displayed while the rectangle moved in a diagonal direction.

3. It might be objected that the failure to obtain a significant interaction between condition and serial position for the normalized errors should preclude specific comparisons on these data. However, the predictions that we made on the basis of the results of Experiment 1 were that (1) the moving/report-direction condition would yield a greater recency effect than would the moving/report-word condition, because task-relevant movement was present in the former but not latter case, and (2) no recency difference would be found between the moving/report-word and the stationary/report-word conditions, because the movement in the former case was task-irrelevant. Given these predictions, we felt that it was appropriate to perform the relevant-specific comparisons on the normalized data, particularly given that the interaction between serial position and condition was significant for the raw errors and marginal for the normalized percentages.

4. We felt that the use of a one-tailed test was appropriate given that there was a clear prediction with respect to the direction of the effect. 
However, the fact that the result was only marginally significant was a bit disappointing. Despite the issue of marginal significance, the data do, we believe, lend credible support to our hypothesis of greater recency with task-relevant chaning-state stimuli. First, the error-rate analyses were as predicted, as was the interaction between serial position and condition for the normalized data. Second, the $t$ test was weakened by the high standard error of the difference between the means that resulted from a peculiarity of using normalized percentages. The normalized percentages for one of the subjects showed a $0 \%$ recency effect in the moving/report-direction condition and a $50 \%$ recency effect in the moving/report-word condition, an effect opposite to our predictions. In fact, that subject had no errors at the last serial position in either condition, made 0 and 4 errors, respectively, in the sixth serial position, and made very few errors in the entire experiment. Due to the low number of errors made overall, each error had a substantial impact on the normal- ized percentages. As a result, the normalized percentages for this subject substantially inflated the standard error of the difference between two means, and therefore diminished the power of the test. When this subject's data were eliminated from the analysis, the difference between conditions was significant $\left[t(40)=2.53, S E_{\overline{\mathrm{D}}}=2.17, p<.01\right.$, onetailed test].

5. At the time that we conducted Experiment 1, we had not yet planned to compare the moving/report-direction condition to the moving-tone condition, and, at that time, 700-msec stimulus durations seemed reasonable for the visual stimuli. When we later designed the moving-tone condition, we decided, after having listened to a variety of candidate stimuli, to use 250 -msec durations for the tones.

(Manuscript received June 6, 1986; revision accepted for publication August 8,1988 .) 